\title{
IMAGE RESTORATION AND ENHANCEMENT USING DEEP LEARNING
}

\author{
Surbhi Sarode, Shunottara Sarade, Sanjana Meshram, Twinkle Kokande, Ashish Palandurkar \\ Department of Computer Science and Engineering, \\ Nagpur Institute of Technology, \\ Nagpur, Maharashtra, India
}

\begin{abstract}
During the process of image acquisition, sometimes images are degraded because of various reasons like low resolution of camera, motion blur, noise etc. This paper presents the work associated with the Image Restoration \& Enhancement techniques. The process of recovering degraded image is known as Image Restoration. Image restoration includes denoising image, image inpainting, etc. Here we proposed Convolution Neural Network (CNN) with Median Filter for removing noise, Region filling Exemplar Based Inpainting Algorithm for image inpainting. Image enhancement is one amongst the problem in image processing. Haze, low lighting etc. are the various problems in images. The aim of Image enhancement is to process an image such that result is more suitable than original image for specific application. Here for haze removal we implement dark channel prior algorithm and for lightning low-light image we proposed functions. Image enhancement improves the appearance of the image.
\end{abstract}

Keywords - Convolutional Neural Network, Dark channel prior, Image enhancement, Region filling exemplar based image inpainting.

\section{INTRODUCTION}

Image restoration refers to recover degraded image. The corruption arises in various forms, like motion blur, low resolution, and noise. Image noise refers to the variations of color and brightness in an image with respect to an ideal image of the real scene. Image noise originates from the atmospheric disturbances. Visually, the noise adds dirty grains with random intensity to the photograph, which in some cases severely degrades visual pleasure and image details. Image noise is ubiquitous due to lack of light, or imperfect camera sensors, Due to cost and space efficiency, mobiles phones are usually shipped with lower-grade camera lenses and sensors. While taking pictures, especially at night time, the resulting images are usually plagued with dirty pixels, which is the image noise. With the increasing usage of mobiles devices, the requirement for an efficient noise-removing algorithm is also increased. Image noise is further categorized by how it is modeled mathematically. There are numerous types of noise such as Gaussian noise, Poisson noise, salt \& pepper noise, etc. Gaussian noise arises during acquiring digital image e.g. sensor noise caused by poor lighting. An image containing salt-and-pepper noise will have dark pixels in bright area and bright pixels in dark area. This kind of noise can be caused by A-to-D converter errors, bit errors in transmission, etc. It can be mostly eliminated by Median filter. Image Inpainting refers to restoration of lost parts of image and reconstructing them based on the background information. It has made wide area within the field of image processing, such as computer graphics, film postproduction, image restoration, image editing. Image enhancement is a process to the sharpening of an image features like edges or contrast to create a graphic display useful for display and analysis. It is used to improve the visual quality. It is also used for improving low level vision applications. The enhancement process does increase the dynamic range of the chosen features in order that they can be detected easily but it does not increase the inherent information content within the data. Image enhancement includes haze removal, lightning low light image, recovery of texture of old image, adjusting contrast etc. The paper contains four sections. 1st section gives introduction the topic whereas 2nd section gives proposed system, section 3 presents conclusion and future scope and last section lists the references.

\section{PROPOSED SYSTEM}

\subsection{Denoising Image:}

In this module, we remove salt \& pepper and Gaussian noise using median filter convolution neural network on a noisy image.

Median Filter:

Median filter is a nonlinear filter which is efficient in removing noise. It is applied to every pixel of image where each pixel is divided into its RGB components and median is assigned to pixel. We need to repeatedly apply the median filter upon the image until all dirty pixels are replaced by the 
median in a fixed-size patch. Patch size should be $(3 \times 3,5 x$ $5, \ldots)$. It removes about $50 \%$ to $90 \%$ noise.

\section{Network Architecture:}

We introduce the Median Filter-CNN denoising algorithm for salt \& pepper, Gaussian noise. In this, we train a patch-based CNN model over training images with artificial noise, and then use the model. Here we used dataset of 5000 images in which we used 4000 images for training and remaining for testing .It has two steps i. pre-processing step ii. CNN training step.

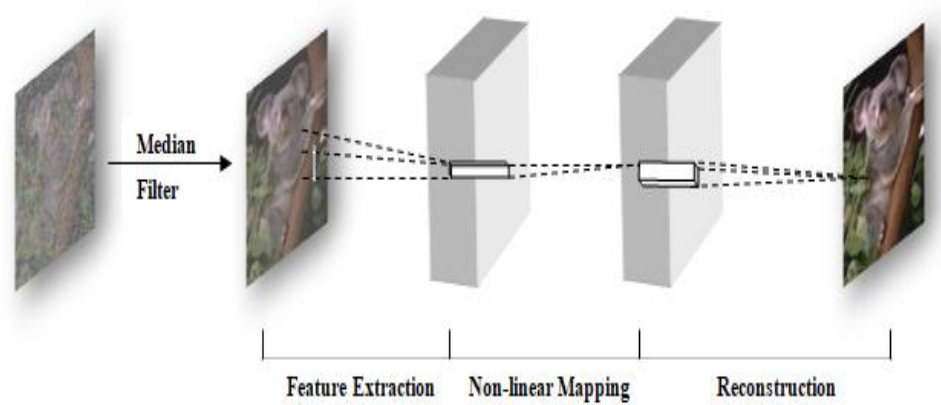

Fig. Network Architecture

i.Preprocessing: We added artificial noise to dataset images. , we pre-process noisy pixels before feed them into CNN training since estimated output cannot be obtained. Thus, we used Median filter in preprocessing.

ii.CNN training:

1. Patch Extraction: The patch of noisy image and clear image is taken.

2. Non -Linear Mapping: The mapping of patch of noisy image is done with patch of clear image.

3. Reconstruction: Here, we reconstruct image and got a denoise image.

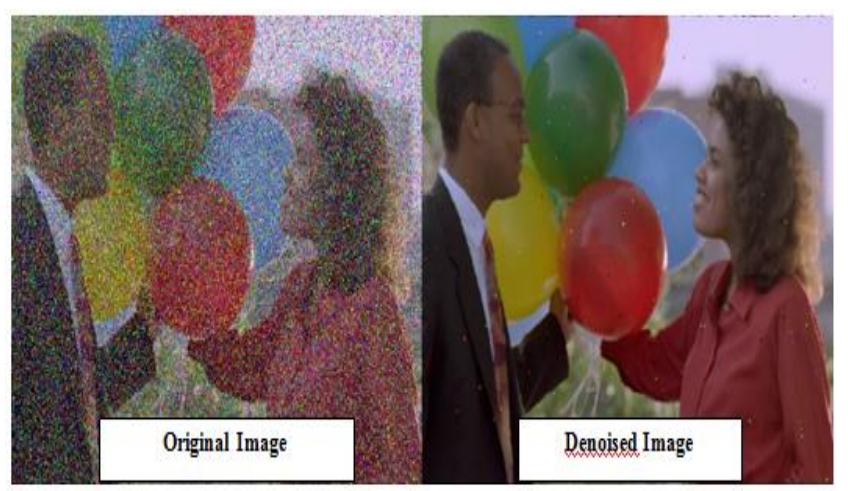

Fig.Denoise Image

\subsection{Image Inpainting:}

Image inpainting is the process of reconstructing missing parts of an image and of reconstructing lost or deteriorated parts of images and videos. This technique is usually accustomed remove unwanted objects from an image. Generally, if wish to get rid of any object from any image via crop, background is also omitted. To get eliminate it we implemented region filling and object removal exemplar based image inpainting algorithm.

\section{Region-filling algorithm Works As Follows:}

1. Extract the manually selected area.

2. Computing patch priorities: This algorithm strongly depends on priority values that are assigned to each patch on the masked image via best first filling strategy. The priority computation is biased toward those patches which: (a) are on the continuation of strong edges and (b) are surrounded by high-confidence pixels.

3. Propagating texture and structure information: Once all priorities on the fill front have been computed, the patch with highest priority is found and fills with it.

4. Updating confidence values: After the patch has been filled with new pixel values, the confidence is updated in the area. 5. Repeat the algorithm until all region is inpainted.

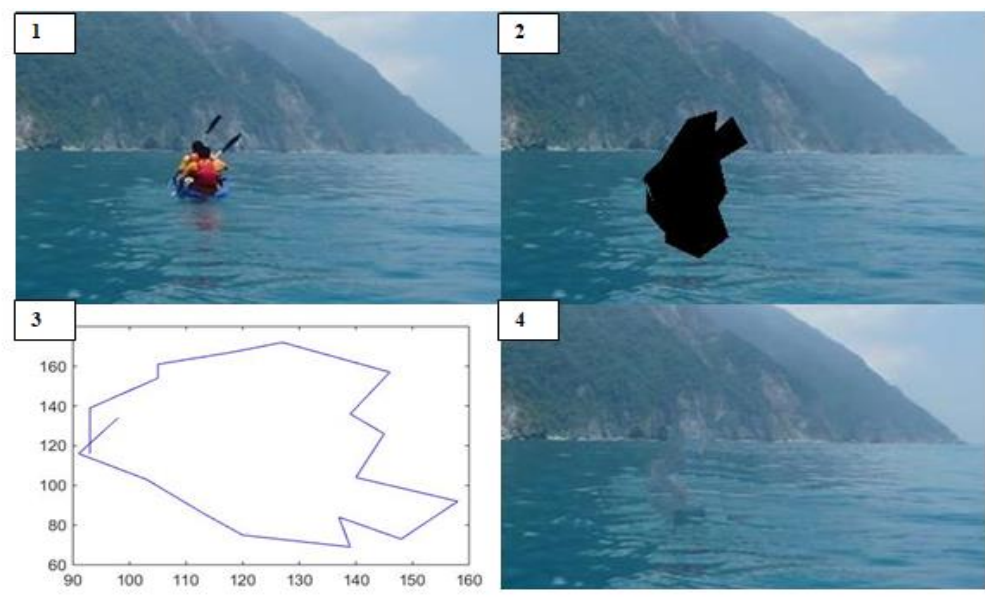

Fig .Image Inpainting

\subsection{Haze Removal:}

When the visibility is less than $1000 \mathrm{~m}$ then it is called haze. Due to weak colors and contrast, appearance of image doesn't seem pleasant thus haze should be removed. It can be achieved by Haze removal algorithm. We implemented Dark channel prior algorithm to get a rid of haze from a hazy image. Any colorful image consist of 3 components RGB i.e Red ,Green, Blue respectively.So,In a hazy image one of this 3 components has less intensity due to which image appears hazy.

The dehazing algorithms are as follows:

1. Estimate the atmospheric light employing a dark channel prior.

2. Estimate the transmission map.

3. Refine the estimated transmission map.

4. Restore the image. 


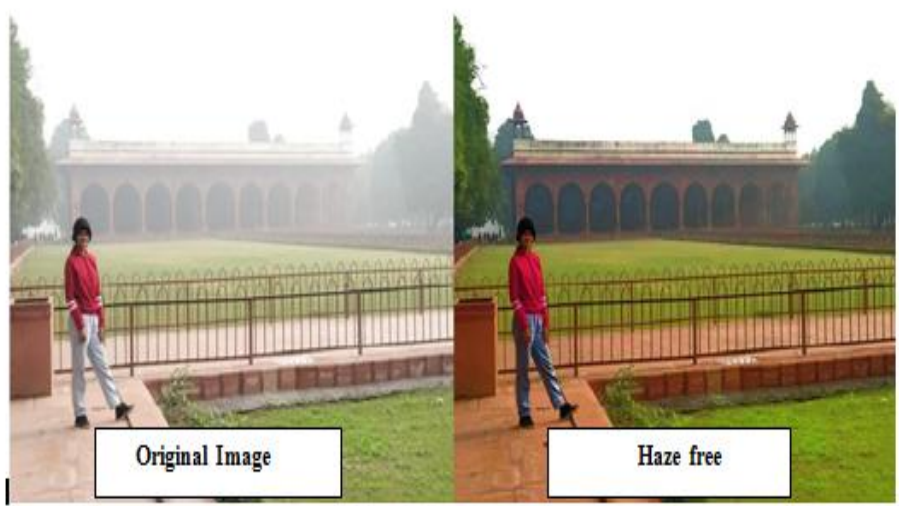

Fig. Haze Removal

\subsection{Lightning Low Light Image:}

Images are degraded due to poor lighting conditions which affect the visual pleasure. We proposed low-light image enhancement to improve the visibility of an image.

Here are the three steps,

Step 1: The low-light image is inverted since white balance of low light image is set at maximum so the low light region appears hazy.

Step 2: Apply the haze removal algorithm to the output of previous step.

Step 3: Invert the results of step 2 to obtain the enhanced image.

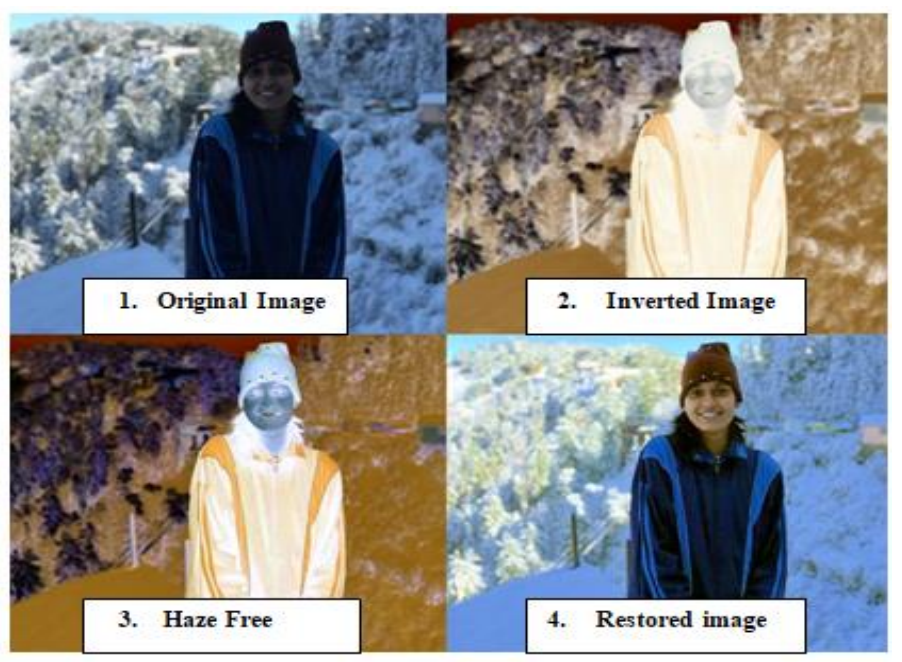

Fig. Lightning Low Light image

\section{CONCLUSION AND FUTURE SCOPE}

This paper presents different modules for restoring and enhancing degraded image. Image noise is generated due to lack of light, lower-grade camera lenses and sensors. Here we proposed Convolution Neural Network (CNN) with Median Filter for removing noise from image. We achieved $80 \%$ accuracy. Image inpainting is the process of reconstructing lost or deteriorated parts of images using information from surrounding areas. Here we proposed Region filling Exemplar Based Inpainting Algorithm. We achieved 87\% accuracy. Image Enhancement is the process of adjusting digital images such that the results are more suitable for displaying or further image analysis like sharpening, etc. In this model, we implemented. Haze removal, Restoring Old Image, Lightning Low Light Image. Haze is a main reason of degradation of outdoor images, weakening both colors and contrasts. Here we used dark channel prior algorithm for removing haze and achieved 96\% accuracy. Images captured in outdoor scenes can be highly degraded due to poor lightning conditions. Here we enhanced low light image and achieved $89 \%$ accuracy.

The images captured by satellite are noisy thus quality of image can be increased by removing noise. Also live video captured while medical checkup of body like sonography consist of noise which might even be removed. Image inpainting can be used for text removal. In foggy region number plates of vehicles isn't visible clearly haze removal algorithm can be implemented. In low lighted areas, if accident occurs then we are able to find the culprit by lightning the low light image or video.

\section{REFERENCE}

[1] Zhang K., Zuo W. (2018) :Ffdnet Toward a fast and flexible solution for CNN based image denoising, IEEE Transactions on Image Processing.

[2] Bhangale S.,Thorat P. (2018): Image Inpainting Using Modified Exemplar- Based Method.In: International Research Journal of Engineering and Technology (IRJET) Volume: 03 Issue: 09.

[3] Bertalmio M., Sapiro V. (2018):Image inpainting. In: Proc.Computer Graphics (SIGGRAPH'00), Singapore, pp. 417-424.

[4] Schechner Y., Narasimhan, S. (2018): Instant dehazing of images using polarization. CVPR, 1:325.

[5] Tang X. (2018): Single Image Haze Removal Using Dark Channel Prior.InIEEE Transactions on Software Engineering 33(12) •

[6] Steffi Agino Priyanka (2018): Low-light Image Enhancement by Principal Component Analysis.In: IEEE Access PP(99):1-1 .

[7] Bo Fu, Xiao-Yang Zhao (2019): A Convolutional Neural Networks Denoising Approach for Salt and Pepper Noise.

[8] Buades A., Coll B. (2019): A non-local algorithm for image denoising. In: IEEE Computer Society Conference on Computer Vision and Pattern Recognition vol. 2, pp.60-65. 
[9] Chen C.,Xiong Z. (2019): "Deep boosting for image denoising," in European Conference on Computer Vision.

[10] Deng L., Huang T., Zhao X. (2019) :Exemplar-Based Image Inpainting Using a Modified Priority Definition..

[11] Perez P. and Toyama K. (2019): Region Filling and Object Removal by Exemplar-Based Image Inpainting.In: IEEE TRANSACTIONS ON IMAGE PROCESSING, VOL. 13, NO. 9.

[12] Ashikhmin M. (2019): Synthesizing natural textures. In Proc. ACM Symposium on Interactive 3D Graphics, pages 217-226, Research Triangle Park, NC.

[13] Hanspa R. (2019): A Survey of Image Enhancement Techniques.

[14] Kuldeep Narayan Shukla(2019): A Review on Image Enhancement Techniques.

[15] Kaiming He, Jian Sun (2019): Single Image Haze Removal Using Dark Channel Prior .

[16] Luming L., Deng S. (2019): Convolutional Neural Network with Median Layers for Denoising Salt-andPepper Contaminations. 